\title{
Orthogonal Control of Gene Expression in Plants Using Synthetic Promoters and CRISPR-Based Transcription Factors
}

\section{Shaunak Kar ( $\sim$ shaunak@utexas.edu )}

The University of Texas at Austin https://orcid.org/0000-0003-3354-1789

\section{Yogendra Bordiya}

The University of Texas at Austin

\section{Nestor Rodriguez}

The University of Texas at Austin

Junghyun Kim

The University of Texas at Austin

\section{Elizabeth C Gardner}

The University of Texas at Austin

\section{Jimmy D Gollihar}

US Army Research Laboratory

\section{Sibum Sung}

The University of Texas at Austin

\section{Andrew D Ellington}

The University of Texas at Austin

\section{Research Article}

Keywords: Synthetic transcription factor, orthogonal promoter, modular cloning, plant synthetic biology

Posted Date: January 3rd, 2022

DOI: https://doi.org/10.21203/rs.3.rs-1194009/v1

License: (c) (1) This work is licensed under a Creative Commons Attribution 4.0 International License. Read Full License 
1 Orthogonal control of gene expression in plants using synthetic 2 promoters and CRISPR-based transcription factors

3

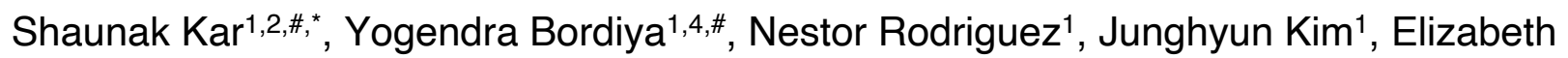
C. Gardner ${ }^{1,2}$, Jimmy D. Gollihar ${ }^{3}$, Sibum Sung ${ }^{1, *}$ and Andrew D. Ellington $1,2, *$

1 Department of Molecular Biosciences, University of Texas at Austin, Austin TX, USA ${ }^{2}$ Center for Systems and Synthetic Biology, University of Texas at Austin, Austin, TX, USA

${ }^{3}$ US Army Research Laboratories-South, Austin, Texas, USA

${ }^{4}$ Present address: Life Sciences Solutions group, Thermo Fisher Scientific, Austin, TX, USA

\# Authors contributed equally

* Corresponding authors

Shaunak Kar: shaunak@utexas.edu

Sibum Sung: sbsung@austin.utexas.edu

Andrew D. Ellington: ellingtonlab@gmail.com

\section{Abstract}

Background: The construction and application of synthetic genetic circuits is frequently improved if gene expression can be orthogonally controlled, relative to the host. In plants, orthogonality can be achieved via the use of CRISPR-based transcription factors that are programmed to act on natural or synthetic promoters. The construction of complex gene circuits can require multiple, orthogonal regulatory interactions, and this in turn requires that the full programmability of CRISPR elements be adapted to non-natural and nonstandard promoters that have few constraints on their design. Therefore, we have developed synthetic promoter elements in which regions upstream of the minimal $35 \mathrm{~S}$ 
33 CaMV promoter are designed from scratch to interact via programmed gRNAs with dCas9

34 fusions that allow activation of gene expression.

Results: A panel of three, mutually orthogonal promoters that can be acted on by artificial

37 gRNAs bound by CRISPR regulators were designed. Guide RNA expression targeting

38 these promoters was in turn controlled by either Pol III (U6) or ethylene-inducible Pol II

39 promoters, implementing for the first time a fully artificial Orthogonal Control System

40 (OCS). Following demonstration of the complete orthogonality of the designs, the OCS

41 was tied to cellular metabolism by putting gRNA expression under the control of an

42 endogenous plant signaling molecule, ethylene. The ability to form complex circuitry was

43 demonstrated via the ethylene-driven, ratiometric expression of fluorescent proteins in

44 single plants.

46 Conclusions: The design of synthetic promoters is highly generalizable to large tracts

47 of sequence space, allowing Orthogonal Control Systems of increasing complexity to 48 potentially be generated at will. The ability to tie in several different basal features of 49 plant molecular biology (Pol II and Pol III promoters, ethylene regulation) to the OCS 50 demonstrates multiple opportunities for engineering at the system level. Moreover, given

51 the fungibility of the core 35S CaMV promoter elements, the derived synthetic promoters 52 can potentially be utilized across a variety of plant species. 


\section{Keywords}

56 Synthetic transcription factor, orthogonal promoter, modular cloning, plant synthetic

57 biology

58

59 Introduction

60

61 The field of synthetic biology aims to revolutionize biotechnology by rationally engineering

62 living organisms (1-6). One aspect of rational engineering is to embed biological

63 organisms with complex information processing systems that can be used to control

64 phenotypes $(2,3,7,8)$, often via synthetic gene circuits that can predictability regulate

65 and tune expression of endogenous as well as transgenes (4, 9-11).

66 However the performance of such synthetic genetic circuits is often plagued by unwanted

67 interactions between the circuit components and the host regulatory system, which can

68 lead to loss of circuit function (10). These unprogrammed interactions can be mitigated

69 via the design and use of genetic parts that have minimal cross-talk with the host, creating

70 orthogonal regulatory or orthogonal control systems (OCS) that can further serve as the

71 basis for constructing complex genetic programs with predictable behaviors. In the last

72 two decades an increasing number of well-characterized genetic parts have been

73 combined in circuits capable of complex dynamic behaviors, including bi-stable switches,

74 oscillators, pulse generators, Boolean-complete logic gates $(7,12-15)$. While OCS and

75 the circuits that comprise them were initially characterized in microbial hosts, more 76 recently a significant fraction of them have been constructed and characterized in 
77 eukaryotic hosts such as yeast and mammalian cells (12,16-19). More recently, synthetic

78 transcriptional control elements have begun to be characterized in plants (20-22).

79 While a variety of artificial plant transcription factors containing diverse DNA binding 80 domains and plant-specific regulatory sequences are known $(20,22)$, orthogonal control

81 requires more programmable DNA binding domains and modular regulatory domains (20,

82 22-24). To this end, we describe an alternate strategy for the construction of orthogonal

83 transcriptional regulatory elements in plants, powered by a single universal transcriptional

84 factor - dCas9:VP64 which has been shown to work in a wide variety of eukaryotic 85 species, including plants $(16,25,26)$. While this transcription factor has primarily been

86 used for the regulation of endogenous genes (25-27), here we describe a generalizable

87 strategy for the universal design and use of synthetic promoters that rely only on the 88 production of specific gRNAs to program dCas9:VP64, and the use of this set of mutually 89 orthogonal promoters for the bottom-up construction of circuits that show multiplexed 90 control of gene expression.

92 Results

93 Design of a modular cloning framework for facile construct assembly

95 Traditionally the process of construction of these synthetic gene expression systems has 96 relied on time-consuming practices of recombinant DNA technology like design of custom

97 primers, PCR amplification, gel extraction of PCR products. Over the last decade the 98 advent of high-throughput cloning techniques, such as Golden-gate cloning with Type IIS 
99 restriction enzymes, has greatly accelerated the design-build-test cycle for the 100 construction and prototyping of synthetic circuits $(7,9,28,29)$. Because the overlaps for 101 assemblies can be modularly specified, multiple parts can be assembled sequentially in 102 a single tube reaction.

103 While a Golden-Gate framework was previously described for the construction of plant 104 expression vectors (30), here we used the highly optimized modular cloning (MoClo) 105 framework, instantiated as a yeast toolkit (YTK) as the basis of our architecture (28). 106 Recently, beyond yeast expression vectors, this framework has been adapted for the 107 construction of a mammalian toolkit (MTK) (9). Along with both YTK and MTK, a plant 108 toolkit based on the YTK architecture will prove essential for seamlessly porting parts and 109 circuits across diverse eukaryotes. Briefly, in this framework the entire vector is divided 110 into particular 'part' types flanked by Bsal restriction sites followed by a unique ligation 111 site. Promoters, genes and terminators are generally categorized into Type 2, 3 and 4 112 parts respectively where each part type has a unique overhang that dictates the 113 compatibility between part types $(9,28)$ (Fig 1A, S1A). This preserves the architecture of 114 each transcriptional unit (promoter-gene-terminator). For the assembly of multiple 115 transcriptional units (TU), each transcriptional unit is first cloned into an 'intermediate' 116 vector flanked by connector sequences that dictate the order of the TUs to be stitched 117 together. By using appropriate connectors, each TU can be further assembled into a final 118 expression vector in a single pot reaction (Fig S1B) [20]. This modular approach enables 119 rapid assembly of increasingly complex genetic circuits comprised of multiple 120 transcriptional units. 
121 Since Agrobacterium-based transformation has been the staple for plant genetic

122 engineering for decades (31), we used compatible vectors as the basis for our framework,

123 and designed and constructed three YTK-compatible shuttle vectors. Each expression

124 vector contains the pVS1 replicon (an Agrobacterium origin of replication - OriV and two 125 supporting proteins - RepA and StaA) and pBR22 origin for propagation in Agrobacterium 126 and E.coli respectively, and a common antibiotic selection cassette (KanR) that has been

127 shown to be functional in both species (Fig 1B, Materials and Methods) (29, 30). The 128 three constructs otherwise differed in the plant selection marker - BASTA, hygromycin, 129 and kanamycin. The resistance markers were expressed from the Nos promoter and also 130 contained a Nos terminator (30) (Fig 1B). The backbone also contains a GFP drop-out 131 cassette that allows easy identification of correct assemblies, which should appear as 132 colonies that lack fluorescence $(9,28)($ Fig 1B).

134 Fluorescence and luminescence reporters are frequently used to study protein 135 localization and interaction in plants and animals (32). To provide these useful reporter 136 parts in the context of our system, we cloned the strong promoter from Cauliflower mosaic 137 virus (35S) as a Type 2 part and its corresponding terminator as a Type 4 part $(33,34)$. 138 These parts can be matched with a number of fluorescent reporter genes (GFP, BFP, 139 YFP and RFP) all as Type 3 parts for robust reporter expression. Combinations of these 140 proteins can also potentially be used for BIFC (Bimolecular Fluorescence 141 Complementation) (35). Similarly, luciferase is commonly used in plant molecular biology 142 to study circadian rhythm (36), test the spatiotemporal activities of regulatory elements 
143 (37), and to study the plant immune system $(38,39)$. Therefore we adapted a luciferase

144 gene from Photinus pyralis, commonly known as firefly luciferase (F-luc) (21).

146 Single TUs comprised of a 35 S promoter, fluorescent reporter genes and the luciferase

147 gene, and a terminator that serves as a polyadenylation signal were assembled into the

148 Agrobacterium shuttle expression vector (Fig 2A-C). The activity of constructs was

149 assayed using transient expression in Nicotiana benthamiana (30). As expected, we see

150 strong activity of the promoter with the expression of the respective reporter genes (Fig

151 2A-C). In order to diversify the promoters used in circuits (and thereby avoid

152 recombination and potentially silencing), we also included a well-characterized promoter

153 from the Ti plasmid that drives mannopine synthase (Pmas) (40-43). When the 35S

154 promoter was swapped with Pmas, similar expression levels of YFP were achieved (Fig 155 2D).

156

157 Development of an Orthogonal Control System (OCS) to regulate transgene

158 expression

159 One of the primary difficulties with using synthetic biology principles and methods to 160 engineer organisms, especially in eukaryotes, is that the functionality of synthetic circuits 161 is often plagued by unwanted interactions of the circuit 'parts' with the underlying 162 regulatory machinery of the host (44). As a particularly relevant example, systems 163 developed in the past for transgene expression caused severe growth and developmental 164 defects in Arabidopsis and Nicotiana benthamiana $(45,46)$. Therefore, it is paramount to 
165 develop regulatory tools to control transgene expression that minimizes the impact on 166 endogenous plant machinery/physiology, while maintaining the modularity and scalability 167 of synthetic approaches in general.

169 A potential solution to this problem is to develop orthogonal 'parts' that of necessity 170 function independently of endogenous regulation by the host. To this end, we set out to 171 develop a fully integrated Orthogonal Control System (OCS) based on orthogonal 172 synthetic promoters driven by an Artificial Transcription Factor (ATF). We started with

173 the deactivated form of the Cas9 protein (dCas9) fused to the transcriptional activator 174 domain VP64 as a highly programmable ATF $(26,27)$. While dCas9:VP64 has previously 175 been shown to upregulate the expression of endogenous genes via specific guide RNAs 176 (gRNAs) that target the promoter region upstream of those genes $(25,47)$, this strategy 177 has not been utilized for the construction of a fully orthogonal system in which custom 178 promoters can be similarly regulated. Here we develop a suite of synthetic promoters 179 (pATFs, promoter for Artificial Transcription Factor) in which each promoter has a similar 180 modular architecture: varying number of repeats of gRNA binding sites followed by a 181 minimal 35S promoter $(33,34)$. This system is inherently scalable, since new binding 182 sites bound by new gRNAs can be built at will. The complete list of parts (promoters, 183 genes and terminators) is provided in Supplementary Table 1.

185 We initially varied the number of gRNA binding sites (3 and 4) upstream of the minimal 18635 S promoter, and analyzed expression of the reporter using transient assay in Nicotiana 
187 benthamiana. Three repeats provided the best expression of the reporter gene without 188 significant background (Fig 3A). The promoter architecture was further assayed for leaky 189 expression by generating pATF:YFP/BFP/RFP constructs and expressing gRNA 190 constitutively in the absence of dCas9:VP64 (Fig 3A). None of these constructs show 191 expression above background (Fig 3B and 3C). However, upon the addition of 192 constitutively expressed dCas9:VP64 cassette to the circuit, induction of reporter protein 193 expression was observed (Fig. 3B and 3C). Each pATF demonstrated comparable levels 194 of expression (pATF1:YFP - 3-fold, pATF3:BFP - 6-fold and pATF4:RFP - 2 fold) 195 compared to that obtained from the regular 35S promoter (6-fold; Fig 2B). The basic 196 features of the pATF and corresponding gRNAs can thus form the basis for the OCS and 197 should allow us to predictably control reporter and other gene circuits. The complete list 198 of assembled OCS circuits is provided in Supplementary Table 2; as the reader will see, 199 OCS circuitry can be organized in terms of increasing complexity and demonstrates how 200 the Design-Built-Test approach can be used to empirically generate ever more 201 substantive plant phenotypes.

202 In order to show that the OCS designs could also function in stable transgenic Arabidopsis 203 thaliana lines, we assembled the OCS 1-1 and 4-1 circuits (Supplementary Table 2; 204 constitutive YFP and luciferase expression, respectively) in an Agrobacterium expression 205 vector containing with a kanamycin selectable marker as described previously. These 206 OCS constructs were successfully transformed into Arabidopsis thaliana plants (Fig 4A). 207 As expected, the OCS 1-1 $\mathrm{T}_{1}$ plants exhibited constitutive YFP expression (Fig 4B) while 208 the OCS 4-1 plants were imaged (as described in Methods) and the constitutive 
209 expression of luciferase was confirmed (Fig 4C, 4D). Thus, the modular circuits 210 assembled function in two species, as infiltrates in Nicotiana and as transgenics in 211 Arabidopsis.

212

213 Inducible gene expression system via the OCS framework 214

215 The ability to precisely regulate the activity of the transgenes/circuit components based 216 on specific input stimuli is a key feature in programmable synthetic circuits $(48,49)$. In

217 order to enable orthogonal control of induction, we designed gRNA expression cassettes

218 to produce functional gRNAs from inducible Pol II promoters. To prevent nuclear export 219 of gRNAs due to capping and polyadenylation, we used the hammerhead ribozyme 220 (HHR) and Hepatitis Delta Virus (HDV) to cleave the 5' and the 3' ends of the gRNA, 221 respectively. This strategy has been previously shown to lead to the expression of 222 functional gRNAs from Pol II promoters, with activity similar to those driven by the Pol III 223 (U6) promoter $(50,51)$.

224

225 To proof the ribozyme processed gRNA constructs, OCS circuits were assembled where 226 gRNAs were either expressed from a U6 promoter (OCS 1-1) or the 35S promoter (OCS 227 1-5), and could subsequently activate the transcription and expression of reporter genes 228 (YFP) (Fig 5A). For both OCS circuits, downstream reporter gene expression was 229 observed, at similar levels (Fig. 5B). The specific levels of gRNA obtained in each case 230 were analyzed using qRT-PCR (Fig 5C and 5D), and as expected the level of gRNA from 
231 the strong Pol II (35S) driven expression was higher than those obtained with the U6

232 promoter while similar levels of reporter expression were observed for both cases, thus

233 demonstrating that this Pol II driven gRNA expression strategy can be effectively used for

234 OCS activation (Fig 5E). For both these constructs the expression of hdCas9 (human

235 codon optimized dCas9) was also confirmed via Western blot analysis (Fig S2).

236 In order to demonstrate that the Pol II-driven gRNAs could be used as part of an inducible

237 OCS we used the well-characterized synthetic EBS promoter containing the EIN3 binding

238 (52), and placed YFP under the downstream control of the ATF (via pATF-1) (Fig 6A).

239 This circuit (OCS1-9) should be inducible by the volatile organic compound (VOC)

240 ethylene, which is produced from its precursor ACC (1-aminocyclopropane-1-carboxylic

241 acid). Time-dependent expression of YFP is observed in response to 10uM ACC

242 induction (Fig 6B). Both the gRNA-1 and YFP expression levels were quantified before

243 and after induction by qRT-PCR, a maximum of 3-fold induction was observed for both

244 cases (Fig 6C and 6D). Thus, this demonstrates that the activity from synthetic promoters

245 can be controlled via the selective expression of the corresponding gRNAs.

\section{Construction of a panel of mutually orthogonal synthetic promoters}

249 Lack of multiplexed control of transgenes has been a major factor limiting the 250 development of synthetic circuits in plants $(5,6)$. Multiplexed regulation in turn requires 251 a panel of mutually orthogonal promoters and control elements that can operate 252 simultaneously $(5,6)$. Our strategy for synthetic promoter design naturally leads to the 
253 generation of expression cassettes that are not only orthogonal to the host but are also

254 mutually orthogonal. The degree of orthogonality can be tuned at will via the sequence 255 design of the multiple gRNA components. By simply minimizing homology between

256 gRNAs, we constructed two additional promoters similar to the architecture of pATF-1, in 257 which gRNA binding sites were followed by a minimal 35 S promoter (pATF-3 and pATF258 4). The orthogonality of these promoters was assayed by assembling expression 259 constructs in which each synthetic promoter controlled the production of a unique 260 fluorescent reporter (pATF-1: YFP, pATF-3: RFP and pATF-4: BFP). The respective 261 gRNAs (gRNA-1, gRNA-3 and gRNA-4) were separately transcribed from a U6 promoter

262 (Fig 7A). When expression constructs were infiltrated into Nicotiana benthamiana, each

263 of the synthetic promoters was specifically upregulated only when its corresponding 264 gRNA was expressed; no background was detected from the remaining two synthetic 265 promoters. (Fig 7B and 7C).

267 Construction of complex ratiometric circuits

269 Now that we have a suite of mutually orthogonal promoters, we sought to construct simple 270 circuits where the activity of each promoter could be independently controlled. Three 271 separate reporter proteins were used to simultaneously monitor the activity of two 272 promoters: pATF-1 with YFP, while both RFP and BFP were under the control of the 273 pATF-3. By leveraging the designed, orthogonal behavior of these promoters it proved 274 possible to construct a ratiometric circuit wherein the activity of pATF-1, and hence YFP 
275 expression, was under the control of ethylene (via ACC), while pATF-3 constitutively

276 drove the expression of RFP and BFP (Fig 8A). As expected, the addition of 10uM ACC,

277 induced the expression of YFP from the pATF-1 promoter (3-fold), while the expression

278 of the other reporters remained constant (Fig $\mathbf{8 B}$ and $\mathbf{8 C}$ ). The ratiometric response was

279 further validated by qRT-PCR; pATF-1 was induced 3-fold following a similar increase in 280 expression of gRNA-1 while there were no changes observed in the transcription of the 281 other two reporter genes (Fig $\mathbf{8 B}$ and $\mathbf{8 C}$ ). The predictable behavior of the designed, 282 artificial control elements in the ratiometric circuit is one of the first examples of complex 283 circuitry to be described in plants, and demonstrates uniquely how natural metabolism 284 and regulatory circuitry can be interfaced with free-standing orthogonal control systems.

287 Discussion

289 Transcriptional orthogonality is one of the bedrocks for circuit construction in synthetic 290 biology, and generally serves as the basis for the bottom-up construction of complex 291 circuitry for predictable dynamics $(7,10,17)$. For eukaryotes the construction of multiple 292 promoter elements is hindered by the typically complex regulatory sequences that lie 293 upstream and within promoters (53-55).

295 The design of synthetic eukaryotic promoters has traditionally implemented a common 296 architecture, where a strong transcriptional initiation region is cloned downstream of 
297 orthogonal DNA binding operator sequences and the latter serve as landing pads for 298 synthetic transcription factors (23). The engineered transcription factors have typically 299 consisted of DNA binding proteins (i.e., prokaryotic DNA binding proteins like TetR, Lacl, 300 LexA and PhIF (56-58)) fused to well characterized transcriptional activation domain like 301 VP64. With the advent of programmable DNA binding proteins like zinc finger proteins 302 and TALEs the repertoire of synthetic promoters greatly increased $(23,24,59,60)$. That 303 said, each new synthetic promoter still requires the construction and characterization of 304 its own unique transcription factor $(23,60,61)$.

305

306 These bottlenecks can be circumvented by the use of the highly programmable RNA307 guided DNA binding protein dCas9 (26). The dCas9 RNP fused to transcription activation 308 domains such as VP64 has been used for the upregulation of endogenous genes in a 309 wide variety of eukaryotic species like yeast, mammalian cells and plants $(16,25,26$, 310 62). Here, we have used adapted this 'universal' transcription factor to control the 311 expression of synthetic and orthogonal promoters without the need of addition of any 312 other factors. Using our modular framework, we were able to quickly design and 313 characterize a panel of mutually orthogonal promoters that could drive the production of 314 a variety of outputs, singly and in parallel, including different fluorescent proteins (GFP, 315 BFP, RFP and YFP) and luciferase.

317 The activities of dCas9 based transcription factors can potentially be controlled by simply 318 regulating the expression of their corresponding gRNAs $(16,17)$, enabling the coupling 
319 of natural and synthetic transcription units, and thus natural and overlaid metabolic

320 responses. Here we have effectively used this strategy to couple ethylene sensing (via

321 known EIN3 binding sites) to synthetic (pATF) promoters. Moreover, by changing the

322 number and arrangement of gRNA binding sites synthetic promoters with different levels

323 of activation can be generated, providing further opportunities for design (63). While it

324 has been previously shown that a panel of minimal plant promoters can be used with

325 natural DNA binding sequences for modulating promoter strengths (20), the addition of

326 completely artificial, synthetic promoters as control elements should create opportunities

327 for increasing the specificity and strengths of engineered promoters.

328 Since our strategy for designing synthetic promoters is generalizable it is likely that even

329 more complex circuits can be built by simply incorporating other transcription factor

330 binding sites, or by changing the regulatory 'headpiece' on the dCas9 element (for

331 example, to a repressor), (64-66).

332

333 The stabilities of genetic circuitry in plants can be greatly modified by silencing and 334 recombination, amongst other mechanisms $(40,41,43)$. In this regard, the artificial 335 promoter elements that we generate can potentially be crafted to avoid repetition (20), 336 and thus to better avoid silencing and recombination. As viable artificial promoter 337 sequences continue to accumulate, they can be compared and contrasted to identify 338 those that are least vulnerable to modification over time. The facile addition of new parts 339 to the standardize toolkit architecture, particularly terminators, will further increase 
340 opportunities to avoid repetition in ways that again go well beyond what is possible by

341 relying on just a few well-characterized endogenous elements alone.

343 The implementation of orthogonal control systems in plants can be used to limit cross-

344 talk between natural and overlaid regulatory elements, allowing more precise response

345 to a variety of inputs, from VOCs to hormones to temperature, water, and nutrients. The

346 use of orthogonal control systems to enable more precise responses to pathogenesis is

347 especially intriguing given the presence of $R$ genes that are specifically responsive to

348 individual pathogens (effector triggered immunity, ETI) (67). The architecture we have

349 developed is fully generalizable, and can potentially be expanded to non-model plants

350 and other eukaryotic species such as yeast and mammalian cells by the use of

351 appropriate transcription initiation regions under the control of similar gRNA sequences

352 binding sites (68).

354 Conclusion

355 The design of synthetic promoters based on gRNAs and CRISPR-based transcription

356 factor (dCas9:VP64) is highly generalizable to large tracts of sequence space, allowing

357 Orthogonal Control Systems of increasing complexity to potentially be generated at will.

358 The ability to tie in several different basal features of plant molecular biology (Pol II and

359 Pol III promoters, ethylene regulation) to the OCS demonstrates multiple opportunities for 360 engineering at the system level. Moreover, given the fungibility of the core $35 \mathrm{~S}$ CaMV 
361 promoter elements, the derived synthetic promoters can potentially be utilized across a 362 variety of plant species.

364 Materials and Methods

365

366 Plasmid design and construction

368 The plant expression vector was generated using the plasmid plCH86966

369 (Addgene\#48075) as the backbone. The lacZ expression cassette was replaced with the 370 GFP dropout sequence (Supplementary Table 2) to make the plasmid compatible with 371 YTK architecture design. All parts described in Supplementary Table 1, were cloned 372 into the backbone pYTK001 (Addgene \#65108). For the individual transcriptional units, 373 the backbone used was pYTK095 (Addgene \#65202) along with the appropriate 374 connector sequences described in Supplementary Table 3. For the design of orthogonal 375 gRNAs, random 20-mers were generated that had a GC content of $\sim 50 \%$, and that were 376 at least 5 nucleotides away from all sequences in the Nicotiana and Arabidopsis 377 genomes. All oligonucleotides and gblocks were obtained from Integrated DNA 378 Technologies (IDT) unless otherwise stated.

379 For the construction of each genetic element namely promoters, coding sequences and 380 terminators, first they were checked for restriction sites for the following enzymes 381 BsmBI, Bsal, Notl and DrallI. The restriction sites in the coding sequences were removed 382 by the use of synonymous codons while the other elements did not contain any of these 
383 restriction sites. The complete list of parts and constructs are provided in Supplementary

384 Table 1. The part plasmids were cloned into a common vector where each genetic 385 element is flanked by Bsa1 restriction sites followed by appropriate overhangs 386 (Supplementary Table 1). For the assembly of both single TU or multi-TU, the following 387 procedure was used: $10 \mathrm{fmol}$ of backbone plasmid and $20 \mathrm{fmol}$ of parts/TUs were used 388 in a 10uL reaction with 1ul of 10x T4 ligase buffer along with 100 units of Bsal-v2 (single $389 \mathrm{TU}$ ) or Esp3I (multi-TU or parts) and 100 units of T7 DNA ligase. The cycling protocol 390 used is: 24 cycles of $3 \mathrm{~min}$ at $37^{\circ} \mathrm{C}$ (for digestion) and $5 \mathrm{~min}$ at $16^{\circ} \mathrm{C}$ (for ligation) followed 391 by a final digestion step at $37^{\circ} \mathrm{C}$ for $30 \mathrm{~min}$ and the enzymes were heat inactivated $80^{\circ} \mathrm{C}$ 392 for $20 \mathrm{~min}$. All constructs were transformed into $\mathrm{DH} 10 \mathrm{~B}$ cells, grown at $37^{\circ} \mathrm{C}$ using 393 standard chemical transformation procedures. The colonies that lack fluorescence were 394 inoculated and plasmids were extracted using Qiagen Miniprep kit according to the 395 manufacturer's instructions Plasmids were maintained as the following antibiotics 396 kanamycin (50ug/mL), chloramphenicol (34ug/mL) and carbenicillin $(100 \mathrm{ug} / \mathrm{mL})$ 397 wherever required. The plasmids were sequence verified by Sanger sequencing (UT 398 Austin Genomic Sequencing and Analysis Facility). The correct constructs were then 399 transformed into Agrobacterium tumefaciens strain GV3101 (resistant to Gentamycin and 400 Rifampicin) and used either for transient expression in Nicotiana benthamiana or to 401 generate stable lines in Arabidopsis thaliana. The following enzymes were used for the 402 assemblies - Bsal-v2 (NEB \#R3733S), Esp3I (NEB \#R0734S) and T7 DNA ligase (NEB 403 \#M0318S). 
406 Nicotiana benthamiana and Arabidopsis thaliana plants were grown in soil at $22^{\circ} \mathrm{C}$, and $40716 \mathrm{hr}$ light period. For transient expression, three weeks old plants were syringe-infiltrated 408 with Agrobacterium tumefaciens strain GV3101 $\left(\mathrm{OD}_{600}=0.5\right)$ and leaves were imaged 409 under Olympus BX53 Digital Fluorescence Microscope or harvested for RNA and/or 410 protein analysis. To create stable transformation in Arabidopsis, floral dip method (69)

411 was used. $T_{1}$ plants were selected on half MS Kanamycin $(50 \mu \mathrm{g} / \mathrm{ml})$ plates and the 412 selected T1 plants were analyzed using an Olympus BX53 Digital Fluorescence 413 Microscope and a NightOwl imager for YFP expression and luciferase expression, 414 respectively. For circuits that constitutively expressed YFP (OCS1-1) and luciferase 415 (OCS4-1) no other obvious phenotypic differences were observed across numerous 416 individual plants.

$418 \quad R N A$ extraction and $q R T-P C R$

419 RNA was extracted using TRIzol reagent (Ambion). $1 \mu \mathrm{g}$ total RNA was used to synthesize 420 cDNA. After DNasel treatment to remove any DNA contamination, random primer mix 421 (NEB \#S1330S) and M-MLV Reverse transcriptase (Invitrogen \#28025-013) were used 422 for first strand synthesis. qRT-PCR was used to quantify the RNA prepared from transient 423 expression experiments. AzuraQuant qPCR Master Mix (Azura Genomics) was used with 424 initial incubation at $95^{\circ} \mathrm{C}$ for 2 min followed by 40 cycles of $95^{\circ} \mathrm{C}$ for $10 \sec$ and $60{ }^{\circ} \mathrm{C}$ 425 for $30 \mathrm{sec}$. Level of target RNA was calculated from the difference of threshold cycle (Ct) 
426 values between reference (5S rRNA) and target gene using at least three independent

427 replicates

428

429 ACC treatment

430 To check the induction of reporter in response to ACC in the plasmids containing 431 pEBS::YFP/RFP/BFP, Nicotiana benthamiana leaves were infiltrated with Agrobacterium;

432 after three days post infiltration, leaf discs were cut using cork borer and incubated in 433 either $0 \mu \mathrm{M}$ or $10 \mu \mathrm{M}$ ACC for four hours. Fluorescence microscopy was used to check 434 YFP expression after induction.

436 Fluorescence and Luminescence imaging

437 Fluorescence microscope images after Agrobacterium mediated transient expression of 438 YFP, BFP, RFP and GFP in Nicotiana benthamiana leaves were taken using an Olympus 439 BX53 Digital Fluorescence Microscope. For this purpose, leaf discs were cut using cork 440 borer from the area which was infiltrated. Images were taken using either 10X objective 441 lens using the default filters for YFP (500/535nm), BFP (385/448nm), and RFP 442 (560/630nm). The UV filter (350/460nm) was used to take GFP images. The exposure 443 and gain setting were kept constant for each filter within each experiment to compare 444 multiple leaf discs (3 to 6). In all the experiments a leaf disc from a leaf which was not 445 infiltrated with Agrobacterium was used as a negative control in order to account for 446 background fluorescence. All experiments were performed at least three times 447 independently as indicated in the Results. 
448 Expression of luciferase was detected using NightOwl II LB 983 in vivo imaging system 449 (https://www.berthold.com/en/bioanalytic/products/in-vivo-imaging-systems/nightowl-

450 Ib983/). Leaves/plants were sprayed with $100 \mu \mathrm{M}$ D-luciferin, Potassium salt (GoldBio 451 \#LUCK-300). After 5 min of incubation, images were taken in the NightOwl II LB 983. 452 Images were captured with a backlit NightOWL LB 983 NC 100 CCD camera. Photons 453 emitted from luciferase were collected and integrated for a 2 min period. A pseudocolor 454 luminescent image from blue (least intense) to red (most intense), representing the 455 distribution of the detected photons emitted from active luciferase was generated using 456 Indigo software (Berthold Technologies).

458 Western blot

459 Total protein was extracted using urea-based denaturing buffer (100 mM NaH2PO4, $8 \mathrm{M}$ 460 urea, and $10 \mathrm{mM}$ Tris- $\mathrm{HCl}, \mathrm{pH} 8.0$ ) and used for immunoblot analysis to check the 461 expression. The proteins were fractionated by $8 \%$ SDS-PAGE gel and transferred to a 462 polyvinylidene difluoride (PVDF) membrane using a transfer apparatus according to the 463 manufacturer's protocols (Bio-Rad). The membrane was treated with $5 \%$ nonfat milk in 464 PBS-T for 10 min for blocking, and then incubated with Cas9 antibody (Santa cruz, 7A9$4653 \mathrm{~A} 3,1: 500)$ at $4{ }^{\circ} \mathrm{C}$ for overnight. After incubation, the membrane was washed three times 466 for $5 \mathrm{~min}$ and incubated with horseradish peroxidase-conjugated anti-mouse (1:10000) 467 for $2 \mathrm{~h}$. The Blot was washed with PBS-T three times and detected with the ECL system 468 (Thermo scientific, lot\# SE251206). 


\section{Declarations}

471

472 Ethics approval and consent to participate

473 Not Applicable

474

475 Consent for publication

$476 \quad$ Not Applicable

477

478 Availability of data and materials

479 The datasets during and/or analysed during the current study available from the

480 corresponding author on reasonable request

481

482 Competing interests

483 The authors declare no competing interests.

484

$485 \quad$ Funding

486 This work was supported by the Defense Advanced Research Projects Agency (DARPA) 487 agreement HR00111820048 to AE and SS. The content of the information does not 488 necessarily reflect the position or the policy of the Government, and no official 489 endorsement should be inferred. This work was also supported by Welch Foundation 490 grant $(\mathrm{F}-1654)$ to $\mathrm{ADE}$.

491 


\section{Authors' contributions}

493 SK, SS and AE conceived of the project. SK designed the framework and the basic 494 elements of OCS with input from EG, JG and SS. SK and YB assembled all constructs. $495 \mathrm{YB}, \mathrm{NR}$ and JK performed all the testing in Nicotiana with input from SS. All authors 496 contributed with the preparation of figures. SK, YB, JK, SS and AE wrote the manuscript 497 with input from all authors.

498

499

500

We would also like to thank the Qiao lab (UT Austin) for providing details regarding the

501 ethylene induction of the OCS constructs.

502

503

504

505

506

507

508

509

510

511

512

513

514

515

516

517

518

519

520

521

522

523

524

525

\section{Acknowledgments}

\section{Supplementary Information includes}

Fig S1: Workflow describing the assembly of single and multiple transcriptional units (TUs) in a plant expression vector; Fig S2: Western blot to analyze the expression of dCas9:VP64 in OCS constructs - OCS1-1 and OCS 1-5

Table S1: List of all genetic parts used for the construction of OCS constructs

Table S2: List of all OCS constructs

Table S3: List of all Addgene plasmids used in this work

\section{References}

1. Tolle F, Stucheli P, Fussenegger M. Genetic circuitry for personalized human cell therapy. Current opinion in biotechnology. 2019;59:31-8.

2. Scheller L, Fussenegger M. From synthetic biology to human therapy: engineered mammalian cells. Current opinion in biotechnology. 2019;58:108-16.

3. Sedlmayer F, Aubel D, Fussenegger M. Synthetic gene circuits for the detection, elimination and prevention of disease. Nature biomedical engineering. 2018;2(6):399-415.

4. Riglar DT, Silver PA. Engineering bacteria for diagnostic and therapeutic applications. Nature reviews Microbiology. 2018;16(4):214-25.

5. Kassaw TK, Donayre-Torres AJ, Antunes MS, Morey KJ, Medford JI. Engineering synthetic regulatory circuits in plants. Plant science : an international journal of experimental plant biology. 2018;273:13-22. 
526 6. de Lange O, Klavins E, Nemhauser J. Synthetic genetic circuits in crop plants. Current 527 opinion in biotechnology. 2018;49:16-22.

528 7. Nielsen AA, Der BS, Shin J, Vaidyanathan P, Paralanov V, Strychalski EA, et al. Genetic 529 circuit design automation. Science (New York, NY). 2016;352(6281):aac7341.

530 8. Medford JI, Prasad A. Towards programmable plant genetic circuits. The Plant journal : 531 for cell and molecular biology. 2016;87(1):139-48.

532 9. Fonseca JP, Bonny AR, Kumar GR, Ng AH, Town J, Wu QC, et al. A Toolkit for Rapid Modular 533 Construction of Biological Circuits in Mammalian Cells. ACS synthetic biology. 2019;8(11):2593534606.

535 10. Brophy JA, Voigt CA. Principles of genetic circuit design. Nature methods. 2014;11(5):508-

53620.

537 11. Mutalik VK, Guimaraes JC, Cambray G, Lam C, Christoffersen MJ, Mai QA, et al. Precise 538 and reliable gene expression via standard transcription and translation initiation elements. 539 Nature methods. 2013;10(4):354-60.

540 12. Weinberg BH, Pham NTH, Caraballo LD, Lozanoski T, Engel A, Bhatia S, et al. Large-scale 541 design of robust genetic circuits with multiple inputs and outputs for mammalian cells. Nature 542 biotechnology. 2017;35(5):453-62.

543 13. Basu S, Gerchman Y, Collins $\mathrm{CH}$, Arnold FH, Weiss R. A synthetic multicellular system for 544 programmed pattern formation. Nature. 2005;434(7037):1130-4.

545 14. Gardner TS, Cantor CR, Collins JJ. Construction of a genetic toggle switch in Escherichia 546 coli. Nature. 2000;403(6767):339-42.

547 15. Elowitz $\mathrm{MB}$, Leibler S. A synthetic oscillatory network of transcriptional regulators. 548 Nature. 2000;403(6767):335-8.

549 16. Kim H, Bojar D, Fussenegger M. A CRISPR/Cas9-based central processing unit to program 550 complex logic computation in human cells. Proceedings of the National Academy of Sciences of 551 the United States of America. 2019;116(15):7214-9.

552 17. Gander MW, Vrana JD, Voje WE, Carothers JM, Klavins E. Digital logic circuits in yeast with 553 CRISPR-dCas9 NOR gates. Nature communications. 2017;8:15459.

554 18. Weber W, Fussenegger M. Engineering of synthetic mammalian gene networks. 555 Chemistry \& biology. 2009;16(3):287-97.

556 19. Kramer BP, Fussenegger M. Hysteresis in a synthetic mammalian gene network. 557 Proceedings of the National Academy of Sciences of the United States of America. 558 2005;102(27):9517-22.

559 20. Belcher MS, Vuu KM, Zhou A, Mansoori N, Agosto Ramos A, Thompson MG, et al. Design 560 of orthogonal regulatory systems for modulating gene expression in plants. Nature chemical 561 biology. 2020;16(8):857-65.

562 21. Schaumberg KA, Antunes MS, Kassaw TK, Xu W, Zalewski CS, Medford JI, et al. 563 Quantitative characterization of genetic parts and circuits for plant synthetic biology. Nature 564 methods. 2016;13(1):94-100.

565 22. Brückner K, Schäfer P, Weber E, Grützner R, Marillonnet S, Tissier A. A library of synthetic 566 transcription activator-like effector-activated promoters for coordinated orthogonal 567 gene expression in plants. The Plant journal : for cell and molecular biology. 2015;82(4):707-16.

568 23. Khalil AS, Lu TK, Bashor CJ, Ramirez CL, Pyenson NC, Joung JK, et al. A synthetic biology 569 framework for programming eukaryotic transcription functions. Cell. 2012;150(3):647-58. 
24. Bae KH, Kwon YD, Shin HC, Hwang MS, Ryu EH, Park KS, et al. Human zinc fingers as building blocks in the construction of artificial transcription factors. Nature biotechnology. 2003;21(3):275-80.

25. Li Z, Zhang D, Xiong X, Yan B, Xie W, Sheen J, et al. A potent Cas9-derived gene activator for plant and mammalian cells. Nature plants. 2017;3(12):930-6.

26. Perez-Pinera P, Kocak DD, Vockley CM, Adler AF, Kabadi AM, Polstein LR, et al. RNA-guided gene activation by CRISPR-Cas9-based transcription factors. Nature methods. 2013;10(10):9736.

27. Chavez A, Tuttle M, Pruitt BW, Ewen-Campen B, Chari R, Ter-Ovanesyan D, et al. Comparison of Cas9 activators in multiple species. Nature methods. 2016;13(7):563-7.

28. Lee ME, DeLoache WC, Cervantes B, Dueber JE. A Highly Characterized Yeast Toolkit for Modular, Multipart Assembly. ACS synthetic biology. 2015;4(9):975-86.

29. Engler C, Youles M, Gruetzner R, Ehnert TM, Werner S, Jones JD, et al. A golden gate modular cloning toolbox for plants. ACS synthetic biology. 2014;3(11):839-43.

30. Weber E, Engler C, Gruetzner R, Werner S, Marillonnet S. A modular cloning system for standardized assembly of multigene constructs. PloS one. 2011;6(2):e16765.

31. Banta LM, Montenegro M. Agrobacterium and Plant Biotechnology. In: Tzfira T, Citovsky V, editors. Agrobacterium: From Biology to Biotechnology. New York, NY: Springer New York; 2008. p. 73-147.

32. Berg RH, Beachy RN. Fluorescent protein applications in plants. Methods Cell Biol. 2008;85:153-77.

33. Benfey PN, Chua NH. The Cauliflower Mosaic Virus 35S Promoter: Combinatorial Regulation of Transcription in Plants. Science (New York, NY). 1990;250(4983):959-66.

34. Odell JT, Nagy F, Chua NH. Identification of DNA sequences required for activity of the cauliflower mosaic virus 35S promoter. Nature. 1985;313(6005):810-2.

35. Kerppola TK. Bimolecular Fluorescence Complementation (BiFC) Analysis as a Probe of Protein Interactions in Living Cells. Annual Review of Biophysics. 2008;37(1):465-87.

36. Tindall AJ, Waller J, Greenwood M, Gould PD, Hartwell J, Hall A. A comparison of highthroughput techniques for assaying circadian rhythms in plants. Plant Methods. 2015;11(1):32.

37. Xiong TC, Sanchez F, Briat J-F, Gaymard F, Dubos C. Spatio-Temporal Imaging of Promoter Activity in Intact Plant Tissues. In: Hehl R, editor. Plant Synthetic Promoters: Methods and Protocols. New York, NY: Springer New York; 2016. p. 103-10.

38. Xu G, Greene GH, Yoo H, Liu L, Marqués J, Motley J, et al. Global translational reprogramming is a fundamental layer of immune regulation in plants. Nature. 2017;545(7655):487-90.

39. Zhou M, Wang W, Karapetyan S, Mwimba M, Marqués J, Buchler NE, et al. Redox rhythm reinforces the circadian clock to gate immune response. Nature. 2015;523:472.

40. Vaillant I, Schubert I, Tourmente S, Mathieu O. MOM1 mediates DNA-methylationindependent silencing of repetitive sequences in Arabidopsis. EMBO Rep. 2006;7(12):1273-8.

41. Matzke MA, Mette MF, Matzke AJ. Transgene silencing by the host genome defense: implications for the evolution of epigenetic control mechanisms in plants and vertebrates. Plant Mol Biol. 2000;43(2-3):401-15. 

specificity of chimeric promoters derived from the octopine and mannopine synthase genes. The Plant Journal. 1995;7(4):661-76.

615 43. Matzke MA, Primig M, Trnovsky J, Matzke AJ. Reversible methylation and inactivation of marker genes in sequentially transformed tobacco plants. Embo j. 1989;8(3):643-9.

617 44. Cardinale S, Arkin AP. Contextualizing context for synthetic biology-identifying causes of 618 failure of synthetic biological systems. Biotechnology Journal. 2012;7(7):856-66.

619 45. Kang H-G, Fang Y, Singh KB. A glucocorticoid-inducible transcription system causes severe 620 growth defects in Arabidopsis and induces defense-related genes. The Plant Journal. 621 1999;20(1):127-33.

622 46. Amirsadeghi S, McDonald AE, Vanlerberghe GC. A glucocorticoid-inducible gene 623 expression system can cause growth defects in tobacco. Planta. 2007;226(2):453-63.

624 47. Lowder LG, Paul JW, 3rd, Qi Y. Multiplexed Transcriptional Activation or Repression in 625 Plants Using CRISPR-dCas9-Based Systems. Methods in molecular biology (Clifton, NJ). 626 2017;1629:167-84.

627 48. Bayer TS, Smolke CD. Programmable ligand-controlled riboregulators of eukaryotic gene 628 expression. Nature Biotechnology. 2005;23(3):337-43.

629 49. Kotula JW, Kerns SJ, Shaket LA, Siraj L, Collins JJ, Way JC, et al. Programmable bacteria 630 detect and record an environmental signal in the mammalian gut. Proceedings of the National 631 Academy of Sciences. 2014;111(13):4838.

632 50. Jacobs JZ, Ciccaglione KM, Tournier V, Zaratiegui M. Implementation of the CRISPR-Cas9 633 system in fission yeast. Nature Communications. 2014;5(1):5344.

634 51. Nissim L, Perli SD, Fridkin A, Perez-Pinera P, Lu TK. Multiplexed and programmable 635 regulation of gene networks with an integrated RNA and CRISPR/Cas toolkit in human cells. 636 Molecular cell. 2014;54(4):698-710.

637 52. Cruz AB, Bianchetti RE, Alves FRR, Purgatto E, Peres LEP, Rossi M, et al. Light, Ethylene 638 and Auxin Signaling Interaction Regulates Carotenoid Biosynthesis During Tomato Fruit Ripening. Frontiers in Plant Science. 2018;9(1370).

640 53. Lelli KM, Slattery M, Mann RS. Disentangling the many layers of eukaryotic transcriptional 641 regulation. Annual review of genetics. 2012;46:43-68.

642 54. Ellwood K, Huang W, Johnson R, Carey M. Multiple layers of cooperativity regulate 643 enhanceosome-responsive RNA polymerase II transcription complex assembly. Mol Cell Biol. 644 1999;19(4):2613-23.

645 55. Chen L. Combinatorial gene regulation by eukaryotic transcription factors. Curr Opin 646 Struct Biol. 1999;9(1):48-55.

647 56. Urlinger S, Baron U, Thellmann M, Hasan MT, Bujard H, Hillen W. Exploring the sequence 648 space for tetracycline-dependent transcriptional activators: novel mutations yield expanded 649 range and sensitivity. Proceedings of the National Academy of Sciences of the United States of 650 America. 2000;97(14):7963-8.

651 57. Bellí G, Garí E, Piedrafita L, Aldea M, Herrero E. An activator/repressor dual system allows 652 tight tetracycline-regulated gene expression in budding yeast. Nucleic Acids Research. 653 1998;26(4):942-7. 
58. Gossen M, Bujard $\mathrm{H}$. Tight control of gene expression in mammalian cells by tetracyclineresponsive promoters. Proceedings of the National Academy of Sciences of the United States of America. 1992;89(12):5547-51.

657 59. Perez-Pinera P, Ousterout DG, Brunger JM, Farin AM, Glass KA, Guilak F, et al. Synergistic 658 and tunable human gene activation by combinations of synthetic transcription factors. Nature 659 methods. 2013;10(3):239-42.

660 60. Li Y, Moore R, Guinn M, Bleris L. Transcription activator-like effector hybrids for 661 conditional control and rewiring of chromosomal transgene expression. Sci Rep. 2012;2:897.

662 61. Cermak T, Doyle EL, Christian M, Wang L, Zhang Y, Schmidt C, et al. Efficient design and 663 assembly of custom TALEN and other TAL effector-based constructs for DNA targeting. Nucleic 664 Acids Research. 2011;39(12):e82.

665 62. Lowder LG, Zhang D, Baltes NJ, Paul JW, 3rd, Tang X, Zheng X, et al. A CRISPR/Cas9 Toolbox 666 for Multiplexed Plant Genome Editing and Transcriptional Regulation. Plant physiology. 667 2015;169(2):971-85.

668 63. Kocak DD, Josephs EA, Bhandarkar V, Adkar SS, Kwon JB, Gersbach CA. Increasing the 669 specificity of CRISPR systems with engineered RNA secondary structures. Nature biotechnology. 670 2019;37(6):657-66.

671 64. Pandelakis M, Delgado E, Ebrahimkhani MR. CRISPR-Based Synthetic Transcription 672 Factors In Vivo: The Future of Therapeutic Cellular Programming. Cell Syst. 2020;10(1):1-14.

673 65. Yeo NC, Chavez A, Lance-Byrne A, Chan Y, Menn D, Milanova D, et al. An enhanced CRISPR 674 repressor for targeted mammalian gene regulation. Nature methods. 2018;15(8):611-6.

675 66. Kwon DY, Zhao YT, Lamonica JM, Zhou Z. Locus-specific histone deacetylation using a 676 synthetic CRISPR-Cas9-based HDAC. Nature communications. 2017;8:15315.

677 67. Gonzalez TL, Liang Y, Nguyen BN, Staskawicz BJ, Loqué D, Hammond MC. Tight regulation 678 of plant immune responses by combining promoter and suicide exon elements. Nucleic Acids 679 Research. 2015;43(14):7152-61.

680 68. Farzadfard F, Perli SD, Lu TK. Tunable and multifunctional eukaryotic transcription factors 681 based on CRISPR/Cas. ACS synthetic biology. 2013;2(10):604-13.

682 69. Zhang X, Henriques R, Lin S-S, Niu Q-W, Chua N-H. Agrobacterium-mediated 683 transformation of Arabidopsis thaliana using the floral dip method. Nature Protocols. 684 2006;1(2):641-6.

685

686 
A

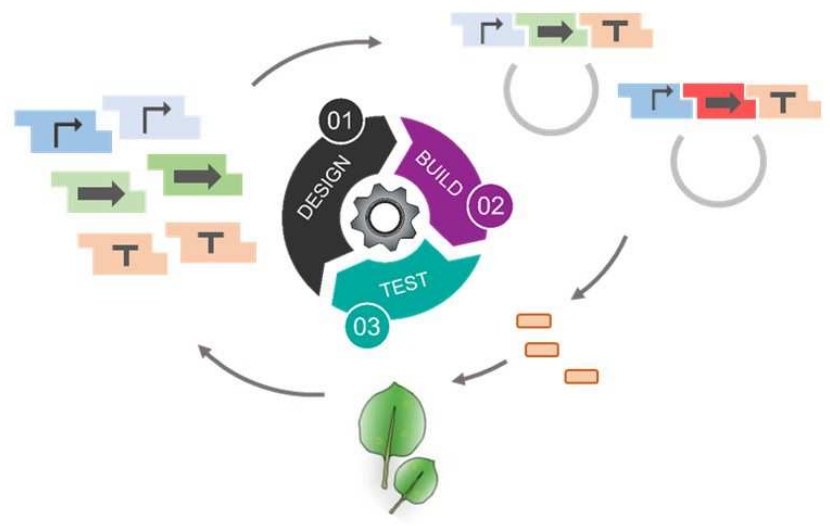

B

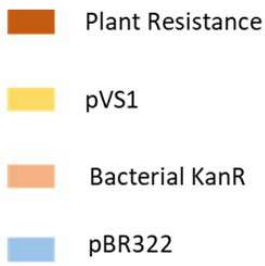

Figure 1. Schematic overview of the design-build-test cycle A. Genetic elements such as promoters, genes and terminators are encoded as modular parts consisting of Bsal recognition sites flanked by specific overhangs to ensure the hierarchical assembly of transcriptional units. Once assembled, the constructs are transformed into Agrobacterium and the reporter expression is characterized in Nicotiana benthamiana leaf infiltrates B. Design of the shuttle vector backbone used for the assembly of constructs and subsequent propagation in Agrobacterium. 
A

A Non-infiltrated
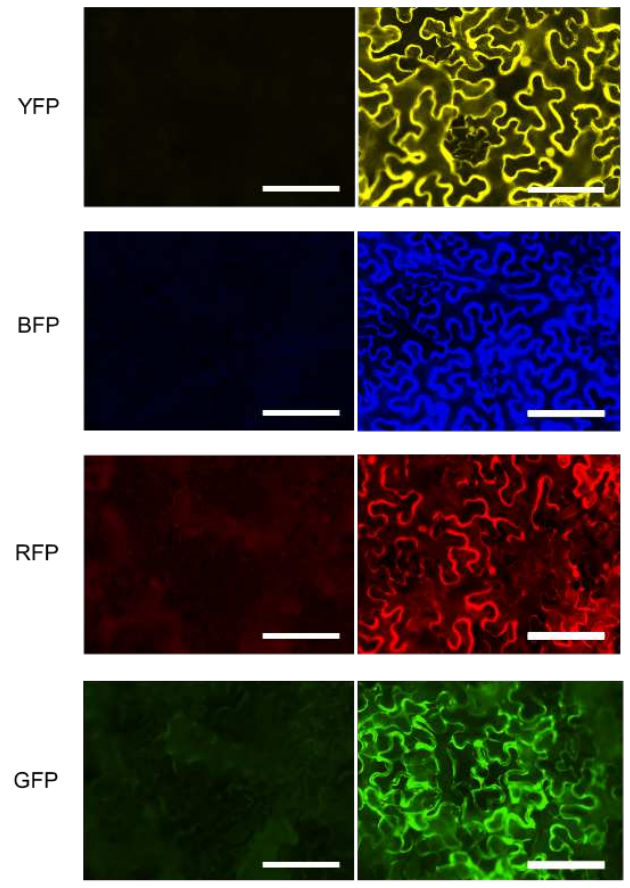

Scale bar : $200 \mu \mathrm{m}$

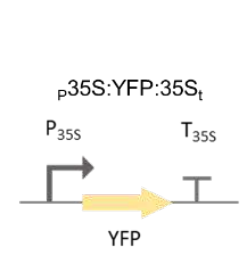

p35S:BFP:35S
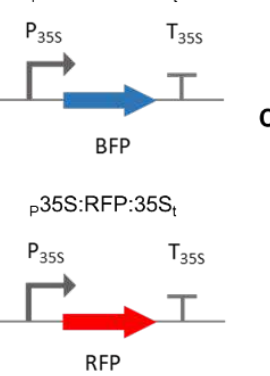

p35S:sfGFP:35S

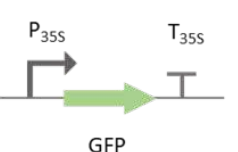

GFP

B

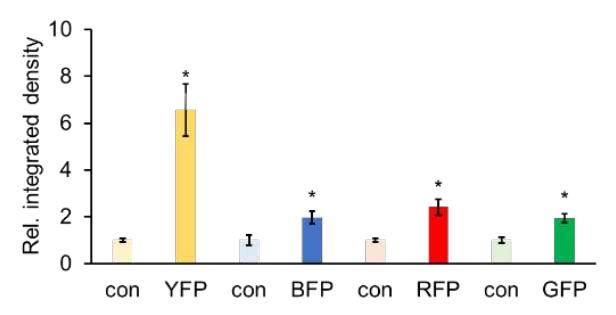

C

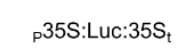

D

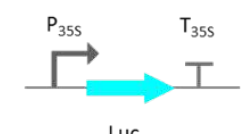

Luc

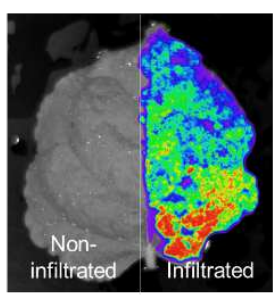

YFP

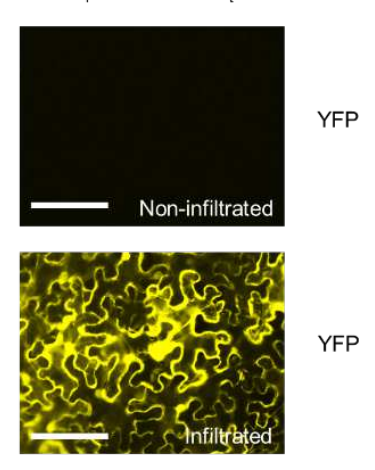

697

Figure 2. Characterization of reporter constructs assembled using APT toolkit. A. Fluorescence microscope images showing Agrobacterium mediated transient expression of YFP, BFP, RFP and GFP under the control of $35 S$ promoter into Nicotiana benthamiana leaves. Images on the left are from non-infiltrated leaves (negative control) captured using the appropriate filter at same exposure and gain settings as was used for the positive images on the right (Material and Methods). B. Relative integrated density of each fluorescence signal (shown in panel A). Integrated density was measured using image $\mathrm{J}$ software and normalized to that of a non-infiltrated control (con). Error bars: S.D. $(n=3$, independent replicates). Asterisks indicate statistical significance in a student t-test $(\mathrm{P}<0.05)$. C. Luminescence reporter luciferase expression shown by Agrobacterium mediated transient expression of luciferase in Nicotiana benthamiana leaves. Left half of the leaf was not infiltrated with Agrobacterium. D. Fluorescence microscope images showing Agrobacterium mediated transient expression of YFP under MAS promoter in Nicotiana benthamiana leaves. Image on the left is the brightfield image for the same construct. 
A
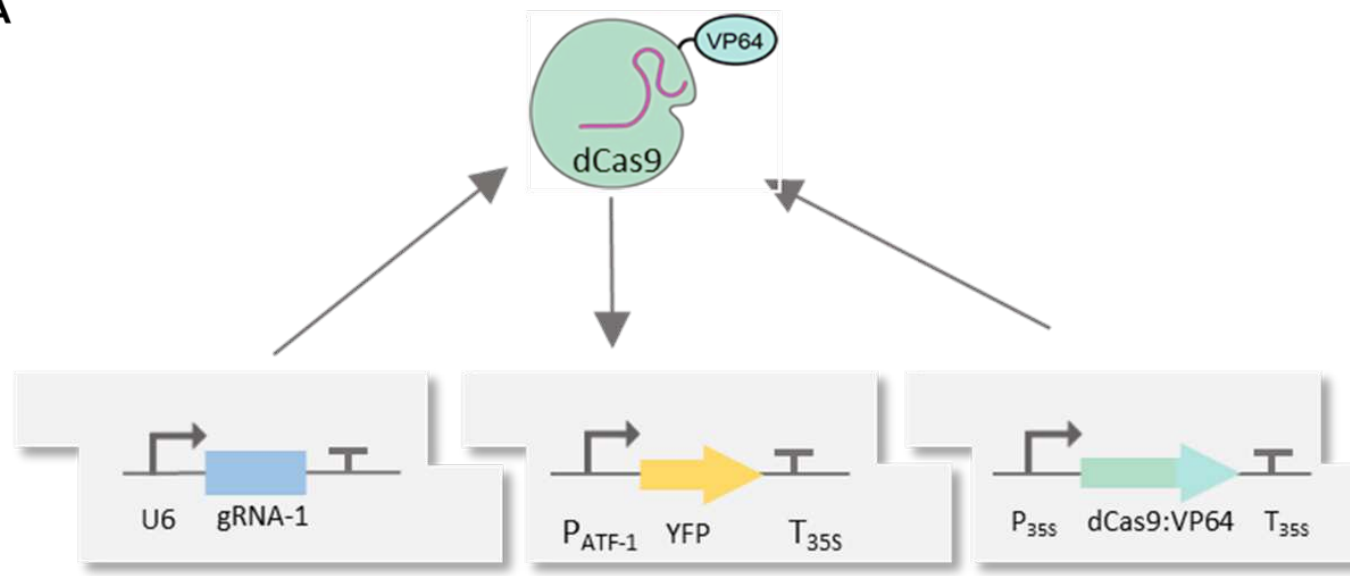

B
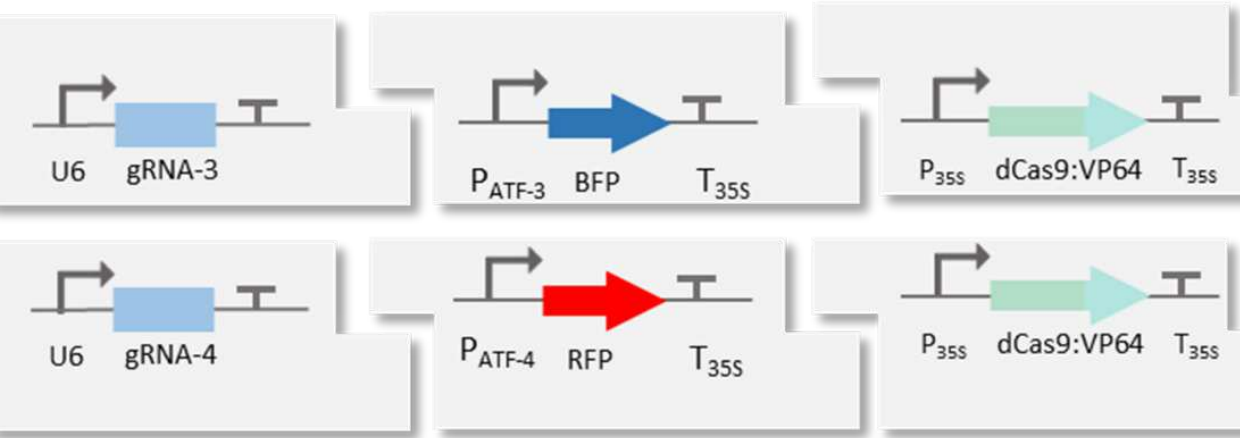

- dCAS9-VP64

+ dCAS9-VP64
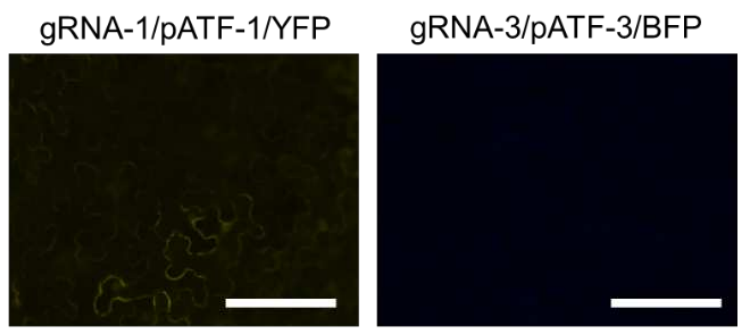

gRNA-4/pATF-4/RFP

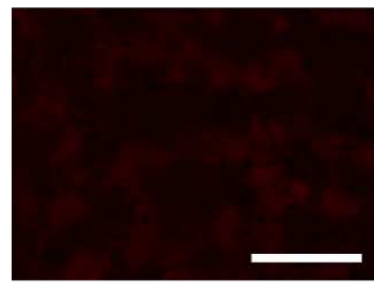

C
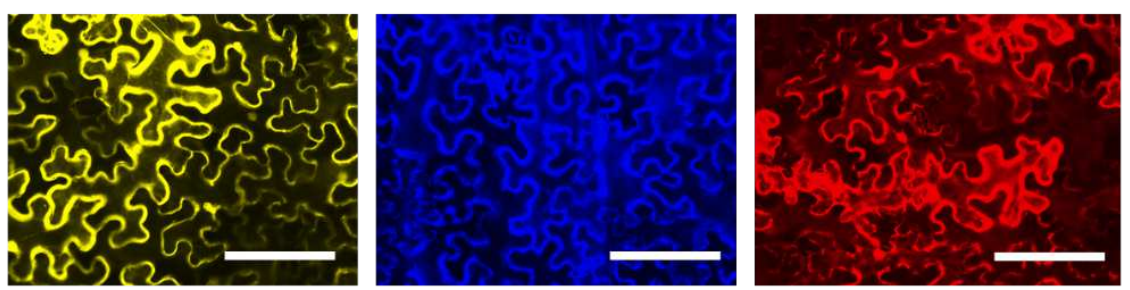

Scale bar : $200 \mu \mathrm{m}$
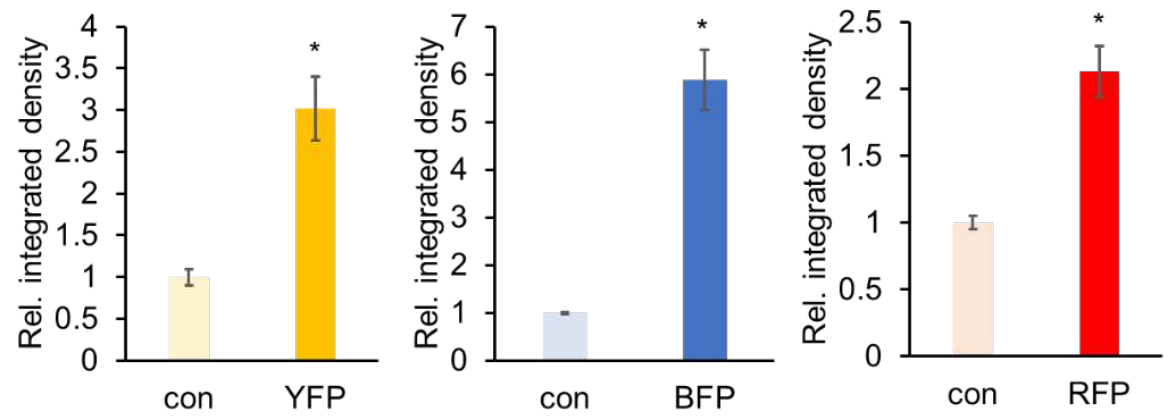
714 Figure 3. Characterization of activity of synthetic pATF promoters. A. Circuit design 715 of dCas9 based artificial transcription factor-controlled activation of synthetic promoters 716 (pATFs). Specific gRNAs are produced by U6 promoter while the expression of the 717 dCas9-VP64 is under the control of the 35S promoter. Reporter genes are under the 718 control of the synthetic promoter (3 repeats of the gRNA followed by minimal $35 \mathrm{~S}$ 719 promoter to the artificial promoter ( $g R N A$ binding site) upstream of a specific fluorescence reporter. B. Fluorescence microscope image showing Agrobacterium mediated transient expression of YFP, BFP and RFP into Nicotiana benthamiana leaves with dCas9-VP64 (bottom panels) and without dCas9-VP64 (upper panels) using three different gRNAs. Images were captured using the appropriate filter (Materials and Methods) at same exposure. C. Relative integrated density of each fluorescence signal (shown in panel B). Integrated density was measured using image $\mathrm{J}$ software and normalized to that of the control (con; - dCAS9-VP64). Error bars: S.D. ( $n=3$, independent replicates). Asterisks 727 indicate statistical significance in a student t-test $(P<0.05)$. 
A

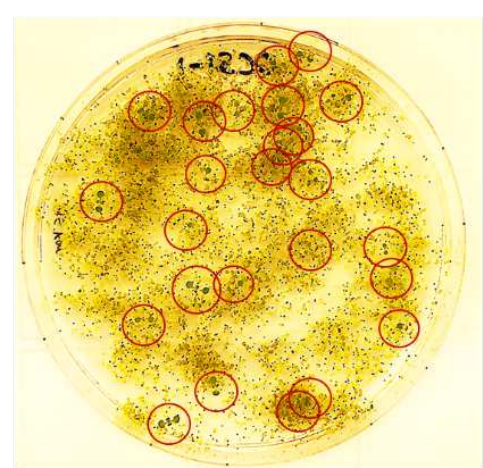

C

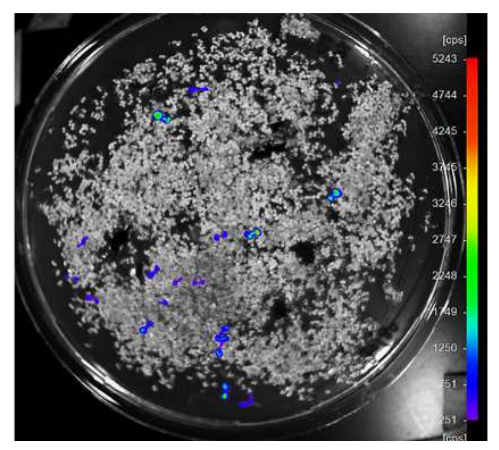

B

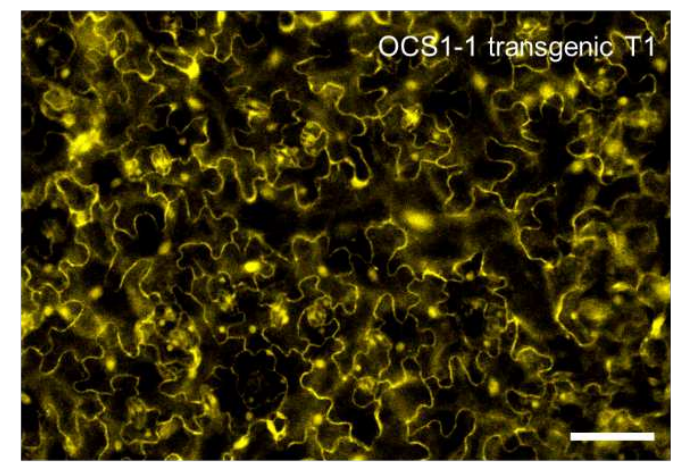

D

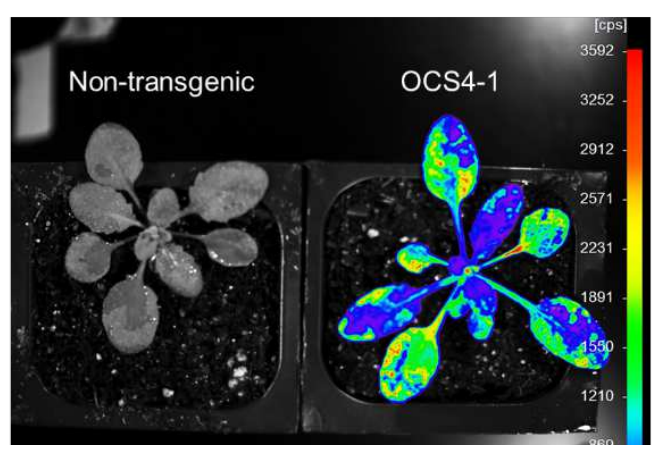

Figure 4. Evaluation of OCS reporter gene expression in transgenic Arabidopsis plants. A. Image showing Kanamycin selection of the transgenic Arabidopsis seedlings on MS media. Seedlings highlighted in the red circle have successfully incorporated OCS circuit. Transformation efficiency is within reasonable ranges $(\sim 1 \%)$ determined by a simple evaluation of the identified seedlings. B. Fluorescence microscope image of Arabidopsis transgenic $\mathrm{T}_{1}$ plants containing the constitutive expression of YFP under the OCS control (OCS 1-1). Scale bar: $50 \mu \mathrm{m}$ C. Image showing Kanamycin selection of the transgenic Arabidopsis seedlings on MS media using luminescence reporter (OCS4-1) taken using the NightOwl (Methods). D. Image of a $\mathrm{T}_{1}$ Arabidopsis plant containing OCS41 at the rosette stage after spraying the luciferin (Methods) containing OCS4-1. This image, taken at the rosette stage using NightOwl after luciferin spray, shows that the luciferase expression is active throughout the adult plant. A non-transgenic plant on the left was used as a negative control in the luminescence reporter assay. 


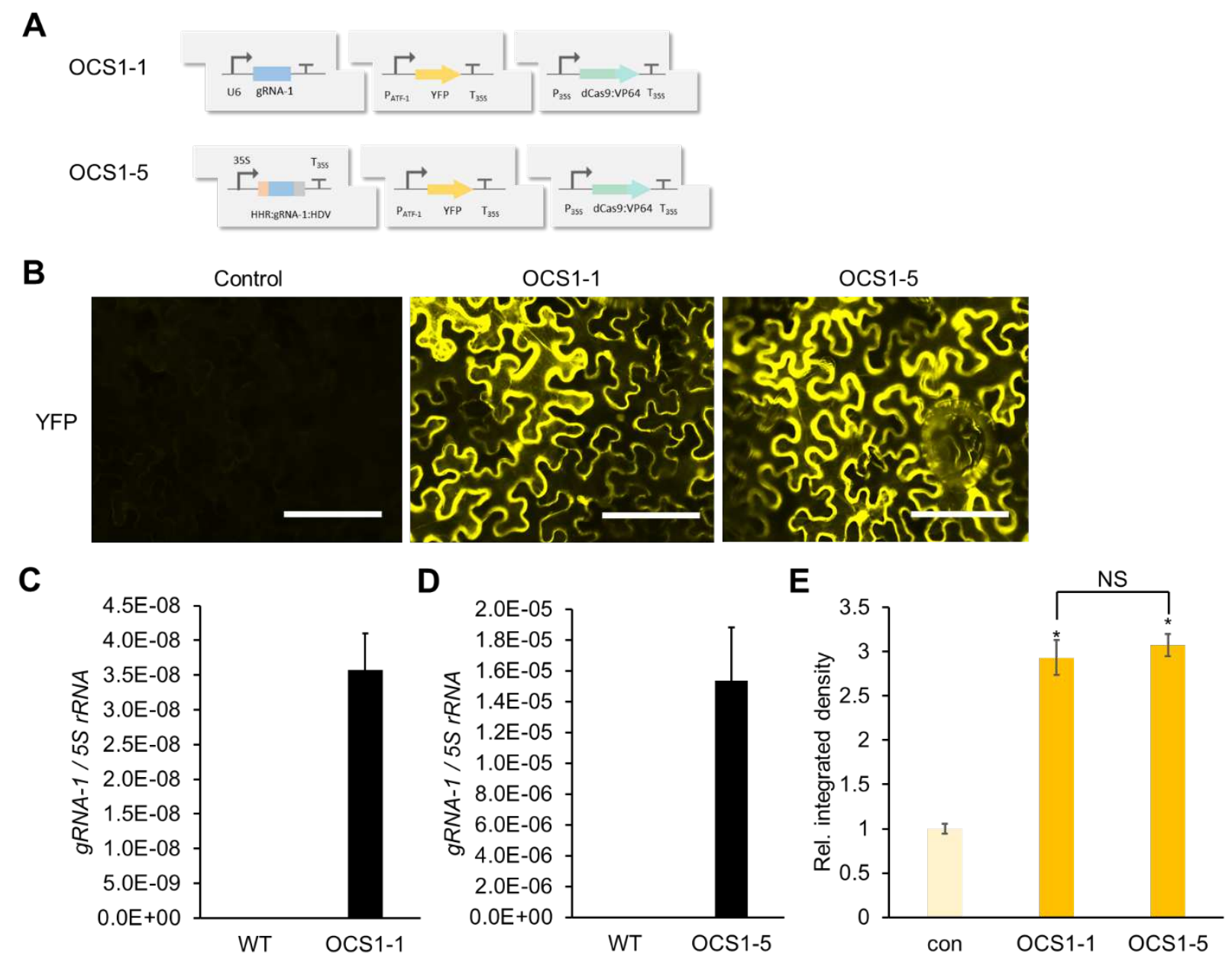

742

743

744

745

746

747

748

749

750

751

752

753

754

755

Figure 5. Design and characterization of gRNA expression modules under the control of Pol II promoters. A. OCS1-1 circuit generates RNA using U6 (Pol III) promoter while OCS1-5 circuit generates gRNA using 35S (Pol II) promoter flanked by self-cleaving ribozymes - HammerHead (HHR) and Hepatitis Delta Virus (HDV). B. Fluorescence microscope images showing Agrobacterium mediated transient expression of OCS constructs with two modalities of gRNA expression (OCS1-1 and OCS1-5). Control images were taken without dCAS9-VP64 expression. Scale bars: $200 \mu \mathrm{m}$ C and D. Quantification of the gRNA-1 expression in OCS constructs (OCS 1-1 (C) and OCS 1-5 (D)) using qPCR relative to $5 S r R N A$. Error bars : S.D. ( $n=3$, independent replicates) E. Relative integrated density of each fluorescence signal (shown in panel B). Integrated density was measured using image $\mathrm{J}$ software and normalized to that of the control (con; - dCas9-VP64). Error bars: S.D. ( $n=3$, independent replicates). Asterisks indicate statistical significance in a student t-test $(P<0.05)$. NS: not significant. 
A

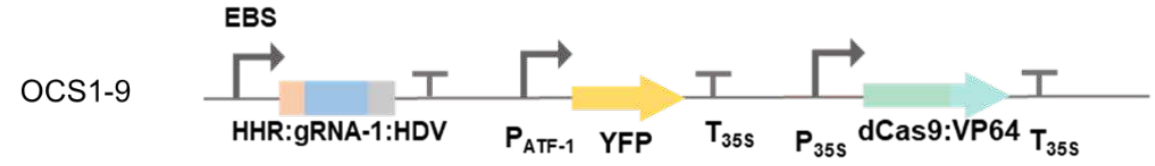

B
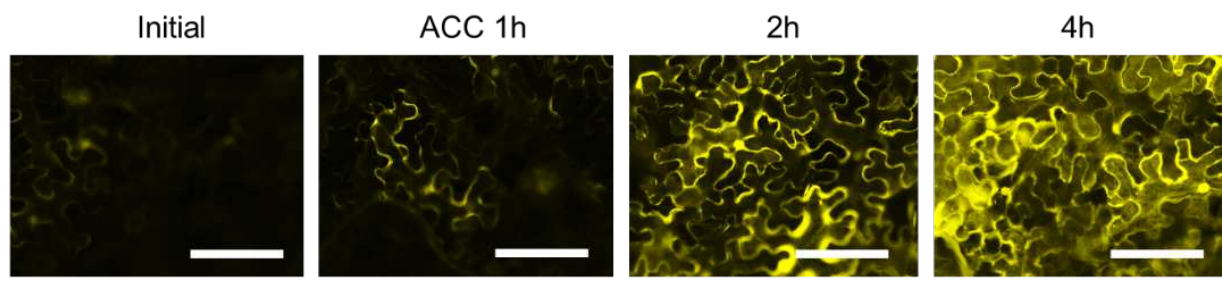

C

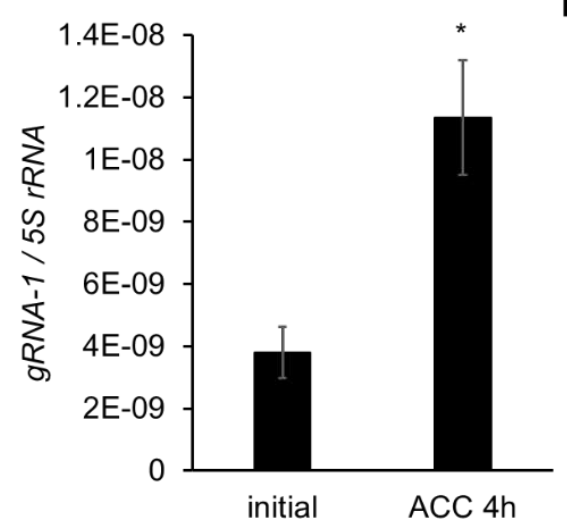

D

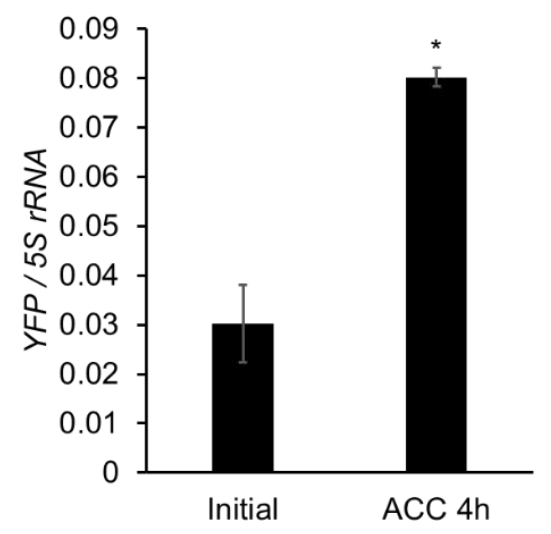

Figure 6. Characterization of an ethylene inducible orthogonal control system. A. OCS1-9 circuit ( $g R N A-1$ is expressed by ethylene inducible EBS promoter) B. Time course fluorescence microscope images showing Agrobacterium mediated transient

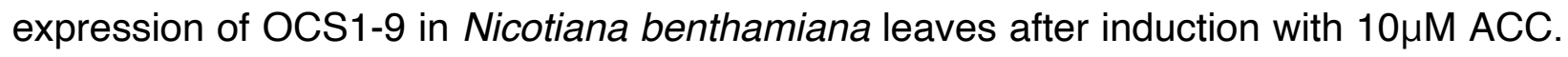

761 Scale bars: $200 \mu \mathrm{m}$ C and D. qPCR quantification of $g R N A-1$ (C) and YFP(D) expression before and after induction with ACC, where both show similar levels of induction demonstrating that the relative change in $g R N A-1$ expression (ethylene induction) results in the differential activation from the pATF-1 promoter. Error bars: S.D. $(n=3$, independent replicates), Asterisks indicate statistical significance in a student t-test $(P<0.05)$. 
A

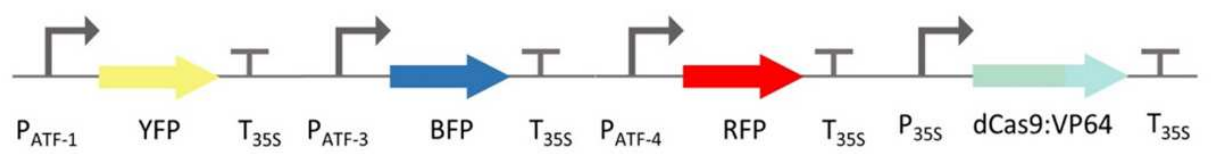

B

(1)

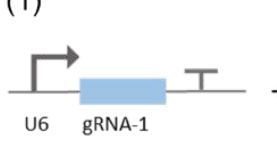

(2)

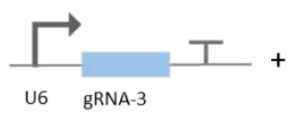

(3)

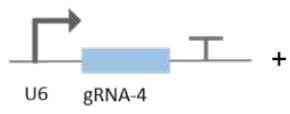

pU6::gRNA-4 pATF1-2::YFP pATF3-2::BFP pATF4-2::RFP 35S::dCas9-VP64
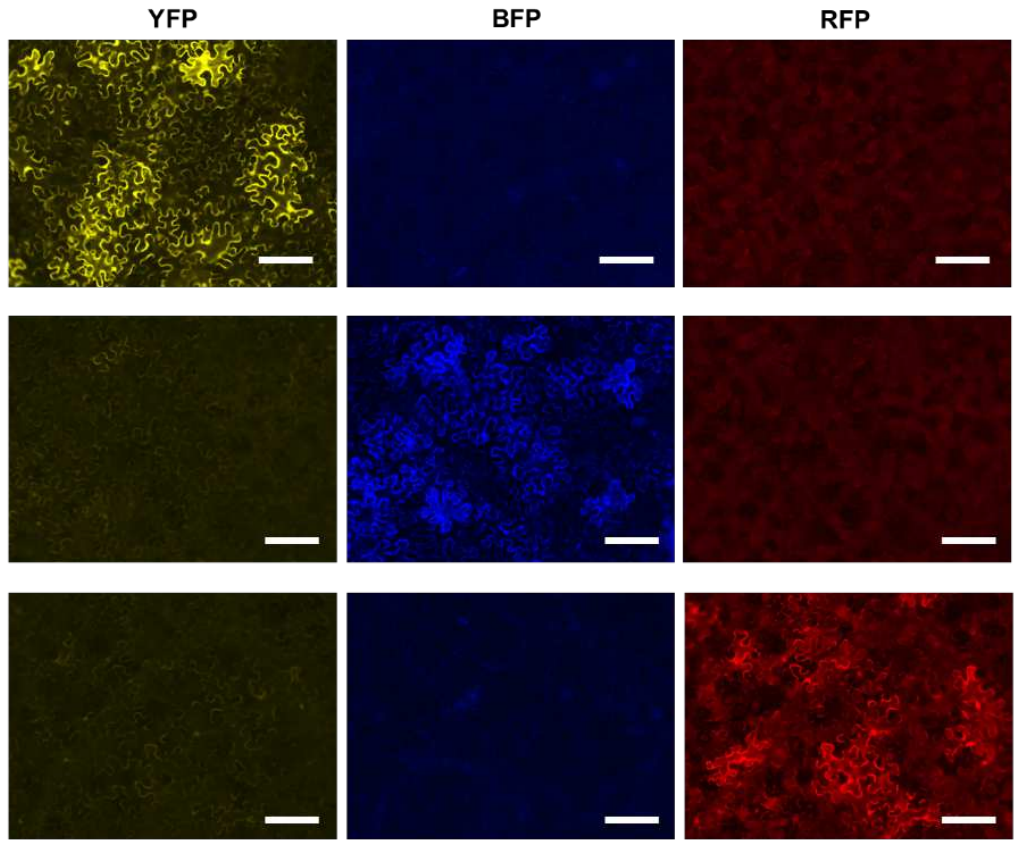

C
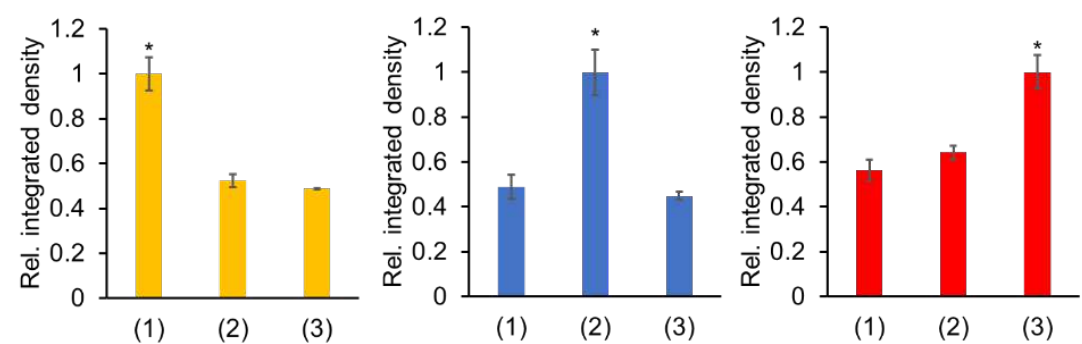

(1) (2) (3)

(1) (2) (3)

Figure 7. Degree of orthogonality of synthetic promoters. A. OCS circuit containing all three synthetic promoters (pATF-1, pATF-3 and pATF-4) driving three different reporter genes namely YFP, BFP and RFP respectively with a single gRNA expressed one at a time under the control of U6 promoter. B. Fluorescence microscope images showing Agrobacterium mediated transient expression of OCS constructs in Nicotiana benthamiana leaves. Scale bars: $200 \mu \mathrm{m}$ C. As observed from the fluorescence images, only the specific gRNA:pATF pair is active, thus demonstrating that the synthetic promoters are mutually orthogonal Relative integrated density of each fluorescence signal (shown in panel B). Integrated density was measured by image $\mathrm{J}$ software and normalized to the highest value. Error bars: S.D. ( $n=3$, independent replicates). Asterisks indicate 777 statistical significance in a student $t$-test $(\mathrm{P}<0.05)$. 
A

ocs3-5

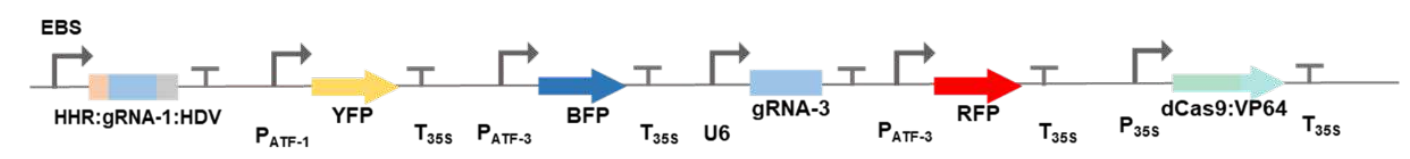

B
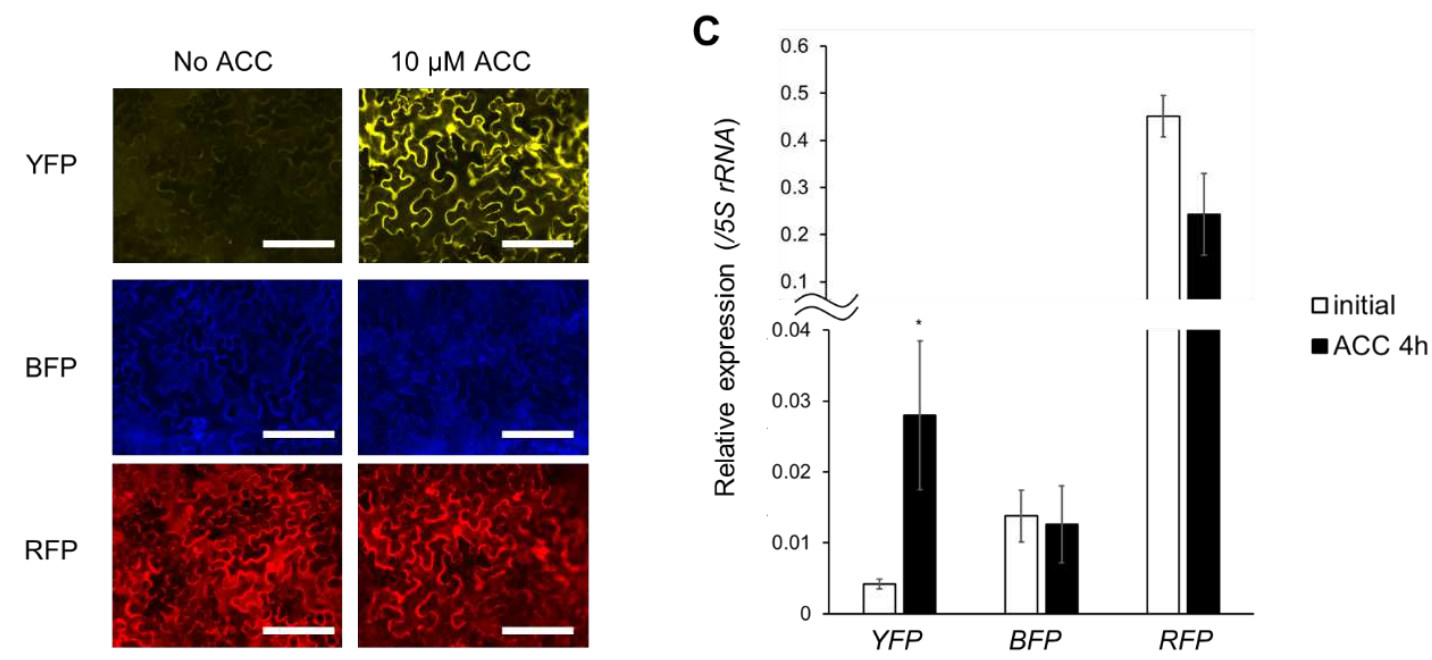

Figure 8. Design and characterization of a ratiometric circuit. A. OSC3-5 contains YFP which is inducible by ACC (pATF-1), while BFP and RFP are constitutively expressed under the control of pATF-3 via the constitutive expression of gRNA-3. B. Fluorescence microscope images showing Agrobacterium mediated transient expression of the ratiometric OCS construct (OCS3-5) in Nicotiana benthamiana leaves with or without $10 \mu \mathrm{M}$ ACC. Scale bars: $200 \mu \mathrm{m}$ C. qPCR quantification of YFP, BFP and RFP shows that YFP is induced after the treatment with ACC while the expression of BFP and RFP remains unchanged before or after ACC induction. Error bars: S.D. $(n=4$, independent replicates). An asterisk indicates statistical significance in a student t-test ( $P$ $<0.05)$. 


\section{Supplementary Files}

This is a list of supplementary files associated with this preprint. Click to download.

- OCSplantsuppV2.pdf

- ocs11.gb

- OCS15.gb

- OCS19.gb

- OCS35.gb

- OCS41.gb

- pAPT.Ex.KanR.gb 